\title{
Recommandations de la Commission interfacultaire médicale suisse (CIMS) concernant les professions médicales et le Service sanitaire de l'Armée
}

\footnotetext{
* La Commission interfacultaire médicale suisse (CIMS) est un groupe de travail des Facultés de médecine et d'autres organisations participant ou s'intéressant aux mêmes tâches et objectifs.
}

Correspondance:

CIMS

Elfenstrasse 18

CH-3000 Berne 16

\section{Introduction}

Les Facultés de médecine et autres institutions membres de la CIMS* (autorités fédérales, FMH, Facultés des sciences, médecins praticiens, étudiants, entre autres), ainsi que des représentants du Service sanitaire et du Groupe du personnel de l'Armée, ont élaboré un concept d'amélioration de l'instruction et des effectifs en médecine militaire et de catastrophe; ils proposent, entre autres, le développement d'une institution fédérale inter-universitaire de formation dans ces deux domaines proches. Ce faisant, la Suisse devrait pouvoir disposer à l'avenir d'un plus grand nombre de médecins formés aux divers aspects de la médecine militaire et de catastrophe. En même temps, ces médecins pourraient combler l'urgent besoin d'un noyau de personnel d'intervention de crise pour des prestations particulières d'assistance en Suisse et à l'étranger.

En synergie avec ce mouvement, le Corps suisse d'aide humanitaire s'intéresse aussi à une coopération dans la formation post-graduée et continue de son personnel médical spécialisé en médecine de catastrophe.

Le développement d'une Académie suisse intégrée de médecine militaire et de catastrophe, conjointement par le Service sanitaire de l'Armée et les Facultés de médecine, est soutenu par la Fédération des médecins suisses, par l'Office fédéral de la santé publique et par les partenaires du Service sanitaire coordonné.

\section{Note préliminaire}

Les professions ou fonctions déclinées ci-après au masculin doivent l'être au féminin et viceversa.

\section{Recommandations}

\section{Service sanitaire et Armée XXI}

1. Encouragement précoce des candidats à une formation dans le domaine de la santé à s'intéresser à une double carrière civile et militaire.

2. Maintien d'un Service sanitaire de qualité fiable dans l'Armée XXI, équivalent à celui d'autres armées comparables, incluant les Services médicaux et pharmaceutiques.

3. Responsabilité du Service sanitaire confiée, dans la mesure du possible à tous les échelons, à des professionnels de la santé. Financement accru de prestations de médecins militaires contractuels ou de médecins civils pour pallier au manque de médecins militaires accomplissant leur service militaire obligatoire ou des services volontaires de milice.

4. Développement d'une Académie suisse intégrée de médecine militaire et de catastrophe.

5. Egalité de traitement du personnel médical ou autre personnel de santé avec celui des autres Armes et Services: suppression de l'obligation de services d'avancement militaire.

6. Les médecins et autres professionnels de la santé qui ne sont pas disposés à effectuer un service d'avancement militaire doivent pouvoir être engagés indépendamment d'un grade pour pouvoir renforcer ou compléter ponctuellement le Service sanitaire de l'Armée. L'actuel système des grades de l'Armée pour le personnel médical doit être révisé dans ce sens, tout comme les conditions permettant d'atteindre le grade d'officier sanitaire. 
7. Possibilité pour les médecins et autre professionnels de la santé d'accomplir jusqu'à un maximum de $75 \%$ des jours de service militaire dépassant les obligations du soldat dans des centres civils de formation ou lors d'engagements formateurs, reconnus les uns et les autres par l'Armée.

8. Réglementation différenciée de la limite d'âge pour les services militaires des médecins et autres professionnels de la santé soumis à de longues formations ou pratiques professionnelles. Une seule limite est fixée à 65 ans pour les médecins militaires et autres professionnels de la santé acceptant d'effectuer des services militaires volontaires.

9. Création d'un modèle de carrière pour les médecins militaires, incluant tous les jours de service en relation avec les études et avec la formation post-graduée.

\section{Coordination et harmonisation de la formation médicale et de l'instruction militaire}

1. Coordination du début et de la durée de l'année académique des Facultés de médecine.

2. Les résultats des examens de fin d'études secondaires devraient être disponibles au plus tard 10 semaines avant le début de l'année académique.
3. A partir de la $3^{\mathrm{e}}$ année d'études, fixation uniforme d'une période libre d'études, d'une durée minimale de 8 semaines tous les deux ans au moins, pour permettre l'accomplissement de services militaires.

4. Coordinations verticale (dans le temps) et horizontale (des contenus) entre les programmes d'enseignement des Facultés et d'instruction du Service sanitaire de l'Armée, incluant la reconnaissance mutuelle des formations.

5. Renforcement, dans les Facultés de médecine, de la fonction de «personne de liaison» avec l'Armée, dans le sens d'un office de contact et de coordination.

6. Enseignement obligatoire de la médecine humanitaire et de catastrophe au sein des Facultés, coordonné avec l'instruction médico-militaire.

7. Institutionnalisation de la Sous-commission CIMS-Armée en tant qu'organe permanent de contrôle et de coordination entre l'Armée et les Facultés. 